\title{
Indicateurs de qualité pour l'éducation relative à l'environnement : une stratégie évaluative ?
}

Michela Mayer

\section{OpenEdition}

Édition électronique

URL : http://journals.openedition.org/ere/6909

DOI : $10.4000 /$ ere.6909

ISSN : 2561-2271

Éditeur

Centr'ERE

Référence électronique

Michela Mayer, «Indicateurs de qualité pour l'éducation relative à l'environnement : une stratégie évaluative ? », Éducation relative à l'environnement [En ligne], Volume 2 | 2000, mis en ligne le 15 septembre 2000, consulté le 17 avril 2021. URL : http://journals.openedition.org/ere/6909 ; DOI : https://doi.org/10.4000/ere.6909

Ce document a été généré automatiquement le 17 avril 2021 


\title{
Indicateurs de qualité pour l'éducation relative à
}

\section{l'environnement : une stratégie évaluative?}

\author{
Michela Mayer
}

1 Parler d'évaluation dans le domaine de l'éducation, c'est parler de quelque chose que l'on pense connaître depuis toujours, et pourtant, c'est seulement depuis les trente dernières années que les activités d'évaluation ont changé de statut : on est passé d'une évaluation considérée comme un jugement entre les mains de qui avait la fonction ou l'autorité pour l'exprimer - l'enseignant, le chef d'établissement, l'inspecteur - à une activité qui consiste surtout à recueillir des données, à décrire, à interpréter et qui est avant tout une activité de recherche, d'approfondissement et de réflexion.

2 Un des risques encourus, le long de ce parcours, est de perdre son temps à défendre des positions que les autres sciences, naturelles et sociales, ont déjà abandonnées: en particulier l'illusion, propre d'un paradigme positiviste, d'une connaissance objective, basée sur des données de fait, sans préalable, et donc en dehors des contextes culturels et des choix de valeurs. Certes, il existe aussi le risque opposé : de renoncer aux responsabilités d'une évaluation au nom de la complexité des processus à évaluer ou d'un relativisme exacerbé.

3 Mais, l'évaluation n'est pas seulement une partie intrinsèque du processus de construction de la connaissance, de la diffusion de nouvelles idées et de nouvelles pratiques innovatrices, elle est aussi un des points de force d'une nouvelle culture de la complexité ; culture qui, d'une part « reconnaît l'interdépendance et la contingence de tous les phénomènes et, par conséquent, leur imprévisibilité intrinsèque » (Mayer, 1999), d'autre part, attribue à l'évaluation des actions et à la prise de conscience rapide des obstacles et des faiblesses, la clé pour une façon d'agir qui, même dans l'incertitude, ne renonce pas à la rationalité. 
Une telle conception de l'évaluation est cohérente avec une conception de l'Éducation relative à l'Environnement (ErE) comme éducation pour le changement, et devient un facteur même de ce changement. Il s'agit de tenir compte des valeurs qui guident les actions, les pratiques éducatives, et d'assumer la tâche de les confronter, de reconnaître qu'il existe des priorités et des intérêts différents; c'est avoir comme objectif non pas l'élimination des diversités, mais leur cohabitation, pour qu'elles évoluent ensemble.

Dans ce contexte, la cohérence entre les hypothèses qui guident des pratiques en ErE et les choix en évaluation est essentielle (Liarakou et Flogaitis, 2000) : on a vu souvent des approches de l'ErE plutôt critiques qui se servent d'instruments d'évaluation de type positiviste, et qui, pourtant, ne peuvent mettre en évidence la profondeur du changement proposé. En effet, les outils de type positiviste sont plus répandus et étudiés, et par conséquent plus faciles à utiliser. Le défi est alors de ne pas tomber dans le piège des stéréotypes et de réexaminer les éléments constitutifs de chaque instrument et les valeurs dont il est porteur, les conditions et la manière dont on pourrait le modifier pour l'adapter aux nécessités d'une évaluation qui s'inspire du paradigme sociocritique.

6 Cet article se propose de contribuer à cette recherche et d'explorer les possibilités que les indicateurs de qualité - instrument qui se retrouve dans le paradigme positiviste et très utilisé dans la recherche éducative internationale - offrent à l'évaluation en ErE. L'analyse a pour but de relever, à partir de l'utilisation ambiguë des termes, les éléments qui, dans le domaine des indicateurs de qualité, semblent plus cohérents et plus intéressants pour une éducation relative à l'environnement orientée vers le changement (Sauvé, 1997). Certains exemples concrets d'indicateurs de qualité, leur utilisation pour l'évaluation et la conduite de projets d'ErE, seront illustrés dans la partie finale.

\section{Une stratégie ambiguë : les indicateurs de qualité}

7 Les indicateurs, que ce soient des indicateurs éducatifs, sociaux ou économiques, sont généralement définis comme des statistiques, simples ou composites, qui traduisent les caractéristiques importantes d'un système et qui ont comme objectif principal «de caractériser la nature du système à travers ses composantes, leurs interrelations, et leur changement dans le temps » (Oakes, 1989).

En ce qui concerne les indicateurs éducatifs, c'est au cours des vingt dernières années que l'intérêt international pour les indicateurs dans l'éducation a augmenté et a amené à une recherche de modèles et de stratégies internationales de collecte de données qui puissent proposer des systèmes d'indicateurs acceptés par plusieurs pays. Les raisons de cet intérêt sont à chercher dans la nécessité :

- de comparer, à l'intérieur d'un même pays, systèmes éducatifs, programmes, écoles, toujours plus autonomes et différenciés ;

- pour les pays industrialisés de passer du problème de la «quantité » - dû à l'exigence des années soixante d'étendre l'enseignement obligatoire à une population en pleine croissance - à la question de la «qualité » de l'enseignement, surtout par rapport à la nécessité de justifier l'investissement public dans l'éducation et de le rendre, au moins, plus productif. 
Les résultats de différentes recherches internationales (surtout les recherches proposées par l'IEA - International Association for the Evaluation of Education Achievement) mettaient, en effet, en évidence la faiblesse des résultats obtenus, surtout en mathématiques et sciences, par plusieurs systèmes éducatifs. Le rapport Gardner sur la situation de l'éducation aux USA, A Nation at Risk: the Imperative for Educational Reform, est de 1983, la même année, le CERI - Centre pour la Recherche et l'Innovation dans l'Enseignement de l'OCDE - approuvait parmi ses différentes activités, un projet spécifique centré sur la "Qualité de l'Éducation» (CERI/OCDE, 1992). Le débat qui suivit, les conférences qui se sont succédées, mettent en évidence d'une part, la nécessité de modèles théoriques et d'informations recueillies sur la base de ces modèles, et de l'autre, la profonde différence de valeurs et de points de vue qui se cachent sous l'utilisation du terme qualité.

Norberto Bottani, responsable du projet sur les indicateurs de qualité de l'OCDE, projet INES (INdicators for Educational Standards), lorsqu'il reconnaît dans la faillite « du projet fondateur de la modernité » une des causes de la crise culturelle et de qualité qui frappe le système éducatif (Bottani, 1989, p. 8), se limite à remplacer l'équation illuministe - plus d'éducation = progrès et meilleure qualité de vie - par une logique pragmatique de marché. L'amélioration de la qualité de l'école serait, selon lui, nécessaire pour sa «double valeur économique»: celle «d'inculquer dans la population un modèle de système économique » et celle de permettre "d'exploiter systématiquement la réserve entière de compétences et d'aptitudes d'une génération " (Bottani, 1989, p. 5).

11 Cette conception de qualité, cohérente avec une conception positiviste et réductionniste de l'éducation, ne peut être partagée par ceux qui se reconnaissent dans le paradigme sociocritique: dans le monde anglais (Elliott, 1993; Norris, 1993) et américain (Guba et Lincoln, 1989), qui déjà allaient dans cette direction, les critiques ne manquent pas. Il n'est donc pas surprenant qu'aux rencontres internationales organisées par l'OCDE à la fin des années 80, diverses conceptions de la qualité soient présentes: de la définition plus orientée vers le marché, c'est-à-dire compétitivité entre écoles et modèles d'enseignement au nom du droit du " client-élève » de choisir l'école qui lui convient le mieux, à celle qui voit la réalisation, à l'intérieur de l'école publique, d'un lieu idéal de rencontre et de confrontation entre les diversités, où les valeurs communes aux contextes social et culturel de chaque pays sont transmises et renforcées.

12 Les « indicateurs de qualité » constituent alors un exemple d'une ambiguïté voulue : on utilise un terme qui vient de la tradition positiviste et qui fait référence à des statistiques, des quantités, des procédures standardisées, et on le met en relation avec un terme qui se réfère à une autre tradition, à d'autres exigences, à un autre ordre des valeurs. La démarche est évidemment celle d'accepter qu'il existe un problème de complexité, mais qu'il faut le simplifier et le réduire : la qualité - en elle-même non mesurable - ne peut être niée, mais elle est représentée, indiquée, à travers la quantité.

Cette démarche peut être inversée et on peut tenter au contraire de "qualifier la quantité ", et d'utiliser les indicateurs comme "indices", comme traces, à l'intérieur d'un système de valeurs cohérent, employant des procédures de médiation et de négociation qui se réfèrent à une évaluation critique et qui implique la participation des différents acteurs. 

épistémologique utilisant des termes qui trouvent leur origine dans un autre langage, sont par ailleurs communs dans notre société comme dans l'éducation relative à l'environnement. Si on réfléchit sur l'utilisation désormais commune d'expressions contradictoires comme "réalités virtuelles ", on se rend compte que quand on parle de « développement durable » ou d'« indicateurs de qualité », on accepte le même genre d'ambiguïté : d'une part, l'utilisation de termes contradictoires reflète un conflit de valeurs qui n'est pas encore résolu, d'autre part, nous devons, ou nous voulons, employer des termes ambigus qui nous permettent de réutiliser ce que nous avions trouvé utile dans un vieux paradigme, à la lumière de l'interprétation que le nouveau nous permet de donner. Comme suggère Robert Stake (1988, p. 51) : « où il y a ambiguïté, où il y a doute, là où le sens n'est pas clair, celui qui apprend a l'opportunité de dépasser celui qui enseigne, le neuf peut dépasser le vieux, on peut ainsi découvrir un sens encore plus profond ».

\section{Quels sont les indicateurs dont nous avons besoin?}

Les indicateurs de qualité lancent un défi à l'ErE : comment concilier deux termes qui semblent opposés, comment mettre ensemble la qualité - des processus, des relations homme-environnement, des relations enseignant-élève - et l'exigence de rationalité et de connaissance, pour prendre des décisions qui concernent notre futur et le futur de notre espèce. La conscience des limites de notre connaissance, de l'imprévisibilité et de l'incertitude en ce qui concerne l'évolution future, nous impose d'évaluer avec le plus grand soin ce que maintenant nous essayons de faire. Et de l'évaluer avec des méthodologies les plus cohérentes possibles avec nos valeurs et nos convictions. Les indicateurs de qualité peuvent-ils offrir une de ces méthodologies?

On compare souvent l'évaluation par un système d'indicateurs à un diagnostic médical, c'est-à-dire à un processus qui, même s'il se base sur des données les plus objectives possibles, est essentiellement un processus d'interprétation, qui, d'une part, se réfère à des modèles et à des théories, et, d'autre part, a besoin d'une large base d'expérience : "L'allusion à un bilan médical convient parfaitement, car elle attire l'attention sur le rôle du praticien appelé à interpréter une information émanant de plusieurs sources, information qui peut contribuer au diagnostic, sans l'imposer, mais ne saurait en ellemême être un jugement » (Nuttal, 1992, p. 18).

L'approche générale proposée par les indicateurs semble donc être cohérente avec ce qu'on demande d'évaluer dans les programmes et les projets pour l'ErE, surtout pour ce qui concerne quelques-unes de ses caractéristiques :

- En premier lieu, recourir à des indicateurs signifie accepter qu'un processus éducatif - que ce soit un projet sur une vaste échelle ou un parcours qui concerne un seul enseignant, une seule classe - soit trop complexe pour être évalué à travers la seule «mesure » des résultats atteints à court terme. Les connaissances acquises par les élèves, les résultats obtenus, sont un aspect seulement, un "indice ", du processus. On a besoin d'autres indicateurs pour comprendre si le processus a été cohérent avec les valeurs (et non seulement les objectifs) qui l'inspirent, comment il peut être amélioré ou quels résultats non prévus on a obtenu.

- Les indicateurs ne se fondent pas nécessairement sur un modèle linéaire input-output, et ils ne sont pas nécessairement des chiffres ou des statistiques: "Dans mon vocabulaire les

Éducation relative à l'environnement, Volume 2 | 2000 
indicateurs représentent seulement une information qui est supposée rendre service à certains, soit pour une prise de décision, soit pour une meilleure compréhension » (Eide, 1989).

- Les indicateurs, en outre, ne peuvent être utilisés seuls: Pour pallier la nature unidimensionnelle de chaque indicateur, il est indispensable de construire un système d'indicateurs, c'est-à-dire un ensemble cohérent d'indicateurs qui donne une bonne représentation de l'état d'un système d'enseignement. Dans l'idéal, un système d'indicateurs renseignera sur la façon dont les différents indicateurs qui le composent travaillent en synergie pour produire un effet global (Nuttal, 1992, p. 17).

-L'approche des indicateurs en évaluation est systémique et, comme pour l'éducation à l'environnement, l'information fournie par un système est plus riche que celle donnée par la somme de ses composantes.

- Un système d'indicateurs doit avoir une logique et une éthique propres, doit être fondé sur un modèle et sur des valeurs qui sont explicitées, et dans lequel l'importance des différents indicateurs est mise en évidence. Un système d'indicateurs de qualité ne peut s'interpréter que « par rapport à des valeurs éducatives et à une expérience scolaire » (Oakes, 1989). Les valeurs et les modèles seront différents selon les contextes culturels et les éléments du projet que l'on veut évaluer, les différences et les similitudes entre modèles - et donc entre les indicateurs - fournissent aussi un instrument de comparaison et d'évaluation.

- Les indicateurs peuvent et doivent être continuellement mis à jour ; le système n'est pas statique, mais dynamique, en évolution continue.

L'approche des indicateurs de qualité semble alors être une approche flexible et extensible, qui, même si elle ne renonce pas entièrement à l'objectivité et à la quantification, en reconnait en même temps ses limites. Les publications de l'OCDE, qui de 1994 à aujourd'hui, mettent à jour les Indicateurs de la Qualité de l'Enseignement pour tous les pays de l'OCDE, ajoutent chaque année de nouveaux indicateurs et de nouvelles mises en garde, en soulignant à la fois la difficulté à recueillir des données vraiment qualitatives et complexes et la nécessité d'une interprétation qui n'isole pas un indicateur des autres indicateurs ou des autres données qui en contextualisent la signification.

\section{Un système d'indicateurs de qualité pour l'ErE}

Quand en 1986 l'Italie décide de participer à la recherche de l'OCDE sur « Environment and Schools Initiatives" - la recherche ENSI (CERI/OCDE, 1991) - je me posais le problème, en tant que coordinateur national, de trouver des critères pour sélectionner les écoles et les initiatives les plus intéressantes. Le choix fût fait, à l'époque, avec l'aide d'un Comité Scientifique national et à travers un questionnaire envoyé à plus de 500 établissements signalés par des associations ou par les autorités scolaires. Le travail que parallèlement proposait l'OCDE sur les indicateurs de qualité, me semblait si intéressant que je proposais à l'ISFOL - Institut pour la Formation au Travail - et avec la collaboration d'une association nationale pour l'environnement - la « Legambiente » une recherche sur «les indicateurs de qualité pour l'éducation relative à l'environnement ».

21 L'objectif déclaré était de chercher des instruments d'évaluation et de comparaison nous étions conscients des limites d'un paradigme réductionniste et positiviste - qui puissent tenir compte de la complexité de la réalité, non seulement de la notion 
d'environnement, mais aussi d'éducation, sans renoncer toutefois à une approche rationnelle. L'ErE était en train d'assumer, à cette époque, un rôle emblématique, de rupture par rapport à l'enseignement des disciplines traditionnelles et obligeait les enseignants à affronter la complexité de notre réalité et de notre façon de la connaître.

Entre sciences de l'éducation et sciences de l'environnement existent, en effet, beaucoup d'éléments communs (Mayer, 1994, p. 91) :

- l'imprévisibilité des résultats : les interactions, les événements casuels sont trop nombreux pour pouvoir envisager des prévisions qui ne soient pas seulement générales et génériques,

- la nécessité, par conséquent, d'une approche globale comportant «des descriptions complexes, holistiques, mettant en jeu une myriade de variables qui ne sont pas fortement indépendantes » (Stake, 1980),

- l'attention aux «émergences", aux structures ordonnées et imprévisibles qui peuvent surgir, même à l'intérieur d'une situation à première vue chaotique,

- la « sensibilité » des questions éducatives et relatives à l'environnement aux valeurs et aux idéologies, la nécessité de comparer, pour les confronter, des valeurs différentes et des représentations différentes de la réalité,

- la difficulté, dans les deux cas, de distinguer le système de l'observateur, et de tenir compte de leur influence réciproque.

Nous étions à la recherche d'un nouveau "paradigme", différent du paradigme positiviste dominant, et le mot "indicateur » nous rappelait le terme "indice » et l'expression "paradigme indiciaire». Le paradigme indiciaire avait été proposé en Italie par l'historien Ginzburg (1986) comme le paradigme de la recherche historique et sociale, en opposition avec le paradigme "galiléen » des sciences naturelles. Dans un paradigme indiciaire, ce sont les différences qui importent et non les éléments en commun qu'on peut généraliser; la rationalité et la rigueur ne se trouvent pas seulement dans la quantification et la généralisation, mais aussi dans l'attention aux indices, aux petits signaux, qui, correctement interprétés, permettent de reconstruire une histoire, d'expliquer des relations, de justifier des faits à première vue inexplicables. Le paradigme indiciaire a des origines anciennes, plus anciennes que la méthode scientifique baconienne ou galiléenne. Ses origines se retrouvent dans les mythes et dans les contes, pour être repris ensuite dans « les Mille et une nuits », par le Zadig de Voltaire, par le frère Guillaume, qui dans « Le nom de la rose » d'Umberto Eco est capable de décrire le cheval de l'Abbé sans l'avoir jamais vu. Actuellement, c'est le paradigme indiciaire qui guide le travail de l'historien de l'art, du détective, du psychologue, mais aussi du paléontologue, du médecin et de l'enseignant qui réfléchissent sur leurs pratiques.

Les indicateurs de qualité nous ont semblé, dès lors, être une méthodologie d'analyse des projets de l'ErE «isomorphe " à la complexité et à la variabilité des projets et cohérente avec une approche sociocritique de l'ErE. Pour ce que ce fût possible, sans retomber dans les indicateurs de performance, le système d'indicateurs que nous avons essayé de construire devait répondre à certaines caractéristiques :

- les indicateurs doivent se référer explicitement à un modèle d'ErE, modèle fondé théoriquement sur des systèmes de valeurs et pratiquement sur des expériences d'ErE,

- ils doivent être organisés en un système, où plusieurs indicateurs sont mis en relations et où les relations constituent une aide à comprendre et à évaluer les projets,

- les indicateurs pour l'ErE doivent être de préférence des indicateurs «composites» « choisir et définir un indicateur composite pour représenter des aspects de l'enseignement, 
c'est reconnaître la complexité de ce dernier» (CERI/INES, 1989, p. 31) - constitués d'autres indicateurs et organisés en « indices ». Les indices doivent être explicités en variables, c'està-dire en éléments qui correspondent à des observations ou à des données, des données d'opinion ou de jugement à recueillir,

- les indicateurs doivent être définis en utilisant soit une méthodologie top down, c'est-à-dire en obtenant certains indicateurs à partir des bases théoriques et des valeurs de départ, soit une méthodologie bottom up, c'est-à-dire en repérant les indicateurs dans des expériences concrètes, avec l'aide des chercheurs. Il ne peut donc exister un seul système d'indicateurs pour tous les projets : on devra toujours tenir compte du contexte, et mettre en place un processus de négociation/collaboration entre évaluateurs et sujets; ce qui assure le respect de la diversité entre cultures, contextes, individus,

- les indicateurs et les indices sont explicités et négociés avant - a priori - et pendant l'évaluation ; ils ne sont pas utilisés seulement pour une évaluation finale, mais aussi comme aide pour la construction et le déroulement des projets, ce qui assure le respect des différents points de vue et la fonction herméneutique de l'évaluation.

En tenant compte de tous ces avertissements, les indicateurs peuvent être utilisés dans l'ErE soit à un niveau micro (le changement individuel ou du petit groupe); soit au niveau meso (le changement au niveau de la structure qui propose le projet d'ErE) et au niveau meta (les changements dans les conceptions de la planète, de la connaissance et de l'éducation).

\section{Construire des systèmes d'indicateurs de qualité comme stratégie d'accompagnement et d'évaluation des projets}

La recherche entreprise en Italie (Mayer, 1991; Mayer et Cogliati Dezza, 1991) présentait un système d'indicateurs de qualité, le modèle sur lequel le système était fondé, et aussi, dans la publication italienne (Ammassari et Palleschi, 1991), les études de cas rédigées par les enseignants qui avaient participé à la recherche.

Le travail, conduit par une équipe composée "d'experts" et d'enseignants qui œuvraient dans des projets d'ErE, avait, durant la première étape, identifié un modèle commun, où toutes les expériences des participants pouvaient se retrouver. Le modèle (figure 1) supposait la nécessité, dans un projet d'ErE, de quatre «aires » fondamentales, reliées les unes aux autres, toutes également indispensables, divisées en étapes :

- une aire existentielle, où le projet doit tenir compte des perceptions et des sentiments, pour arriver à expliciter les valeurs que sous-tendent les actions, et à réfléchir sur la cohérence entre valeurs et comportements ;

- une aire cognitive, où le projet se caractérise par une approche systémique, par le passage du local au global et par la prise en compte de la complexité, non seulement des problèmes, mais aussi des solutions, qui ne peuvent jamais être seulement techniques, mais fondées sur des valeurs;

- une aire opérative, où il est indispensable de travailler sur le terrain, de sortir de l'école et d'engager la communauté, pour arriver au partenariat et à l'action pour l'environnement ;

- une aire méthodologique, observer les approches et les comportements des enseignants qui doivent eux-mêmes être cohérents avec les valeurs qu'ils partagent. Les caractéristiques 
identifiées dans cette aire étaient: la "transversalité » du projet qui ne correspond pas nécessairement à l'interdisciplinarité, mais souligne l'attention prioritaire accordée au problème global et à sa complexité, la «flexibilité des parcours » éducatifs, pour arriver à la « recherche-partagée » entre enseignants et élèves, où les enseignants savent guider une réflexion qui permet d'approfondir des conceptions, d'expliciter des valeurs, de renforcer des comportements et d'envisager de nouvelles actions.

Les indicateurs identifiés n'étaient pas des données statistiques, mais des critères, qui mettaient en évidence la cohérence du projet avec le modèle. Ils étaient " composites ", formés de plusieurs indices et reliés dans un système qui permet d'identifier leurs relations, leur synergie et leur cohérence.

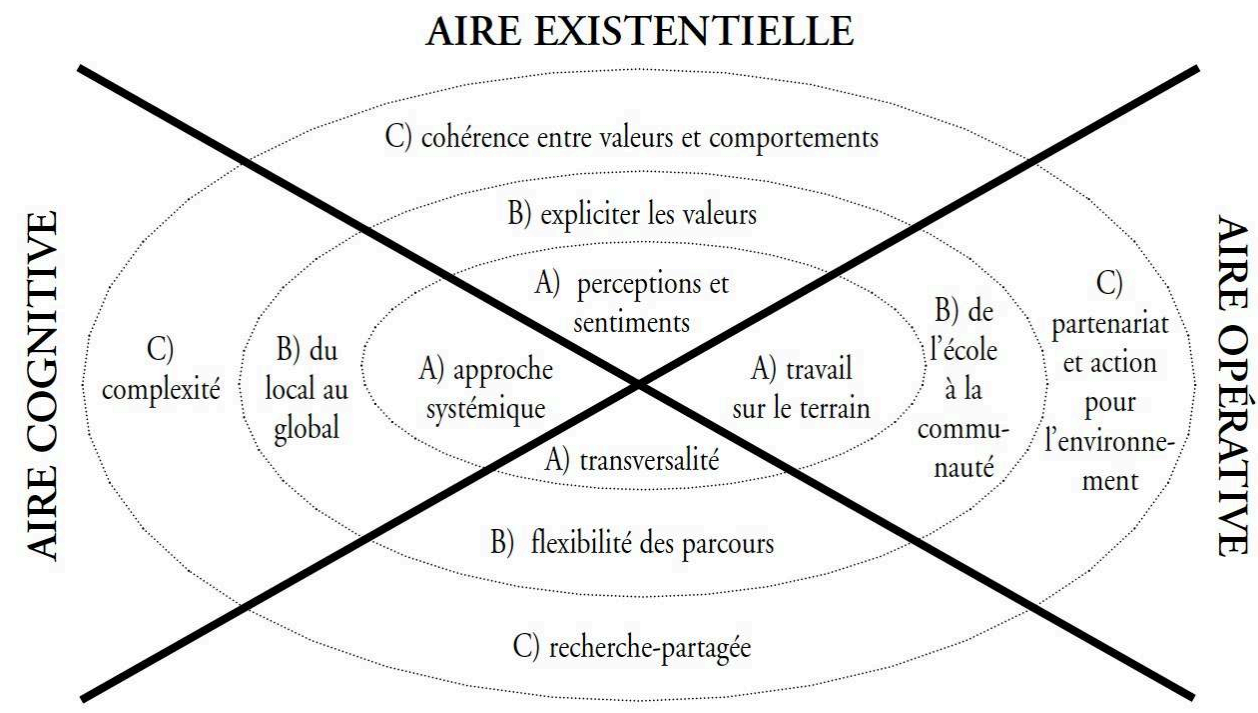

\section{AIRE MÉTHODOLOGIQUE}

Figure 1 : Modèle de projet en éducation relative à l'environnement

Ils étaient regroupés autour de trois critères plus généraux, appelés "indicateurs de champ ", qui correspondaient aux différentes composantes du projet éducatif (figure 2) :

- autour du premier - importance et efficacité du projet au niveau local - nous avons regroupé les indicateurs qui décrivent des conditions d'intervention efficace, c'est-à-dire le travail sur le terrain, l'intégration école-communauté locale, la complexité de l'approche et du parcours éducatif ;

- autour du deuxième - innovation éducative - nous avons considéré comme descripteurs de l'innovation demandée par les projets d'ErE, la transversalité, le changement dans la structure scolaire, la recherche partagée entre enseignants et élèves ;

- autour du troisième - le parcours du changement - nous avons proposé d'observer les changements - des conceptions, des attitudes, des valeurs, des comportements - obtenus chez les élèves, mais aussi la flexibilité du parcours proposé, la mise en valeur des différences, le développement des qualités dynamiques, c'est-à-dire la capacité d'assumer des responsabilités, de prendre des décisions, d'agir de façon autonome. 


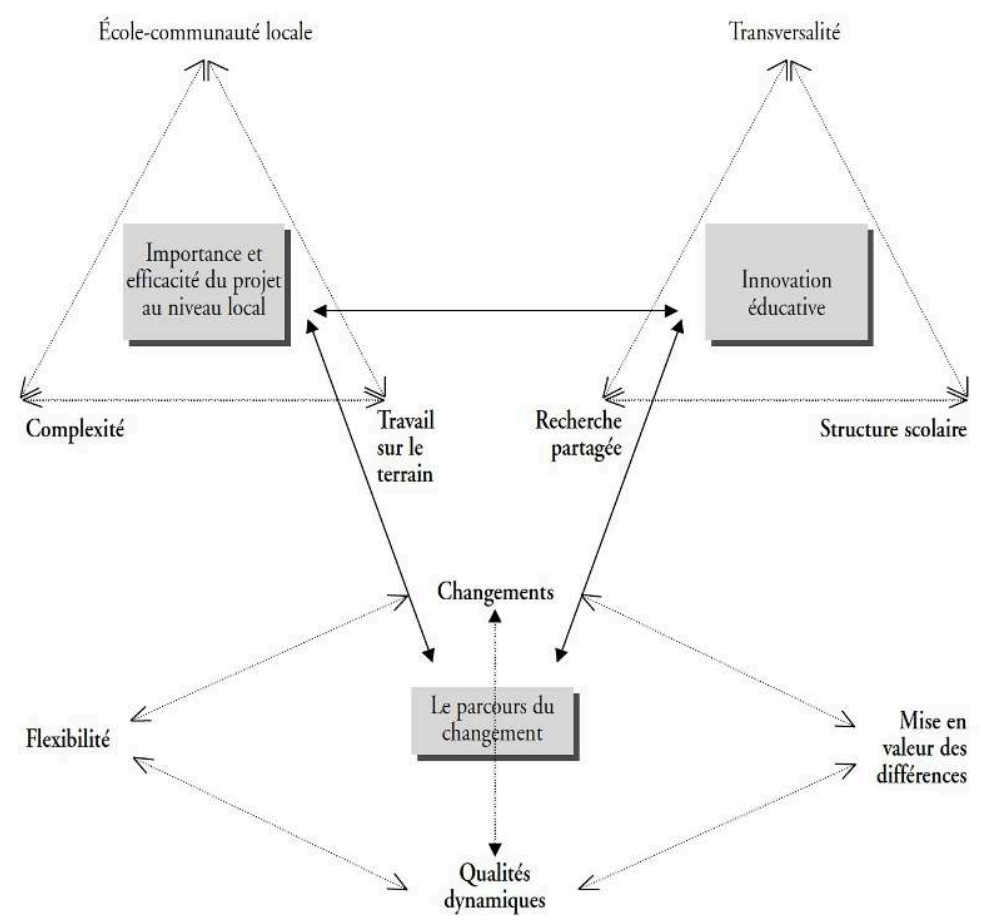

Figure 2 : Le système d'indicateurs

Tous les indicateurs avaient été articulés en indices, pour chaque indice nous avons formulé plusieurs questions : il en a résulté un questionnaire, adressé aux enseignants, dont on a cherché à vérifier la validité en le comparant avec les études de cas que ceuxci avaient préparées dans leurs projets. L'information obtenue du système d'indicateurs était supérieure à celle de leur addition, les études de cas ont, cependant, été préférées au questionnaire comme instruments d'évaluation et de communication de l'expérience.

Notre proposition d'un système d'indicateurs a été largement acceptée, à notre connaissance pas seulement en Italie, et le système semble, au cours des années, avoir maintenu sa validité comme guide pour la construction de nouveaux projets et comme terme de référence pour les enseignants.

\section{Un instrument pour l'auto-évaluation des projets d'ErE}

La recherche italienne était inspirée par les valeurs et les objectifs du projet international ENSI, proposé en 1986 par le CERI-OCDE, et quand en 1990, le projet ENSI a proposé une deuxième phase, le système d'indicateurs de qualité a été présenté aux enseignants italiens qui participaient au projet. Le système n'était, dès lors, plus le résultat d'une construction collective, d'un travail de recherche participative, mais un " a priori », une ressource pour aider les enseignants à construire leur propre projet et leur propre recherche. Dans le projet ENSI, en effet, les enseignants étaient invités à utiliser la recherche-action comme stratégie principale de réflexion sur leur pratique et l'évaluation de leurs projets. «La recherche-action est partie intégrante d'une logique d'élaboration des programmes autres que celle, plus familière, de la planification par objectifs » (Elliott, 1995). 

de mener une réflexion systématique sur leur pratique pédagogique, et de formuler, en utilisant la méthodologie de la recherche-action, des jugements et des évaluations, sur ces pratiques.

\section{démarches principales :}

- l'identification des problèmes de recherche: quels sont les points principaux que nous voulons explorer, et pourquoi?

- la collecte des données - traces, signes, indices - en ayant comme critères la multiplicité/ diversité des techniques (observations, questionnaires, interviews, journal personnel, journal de classe, collection des faits «inattendus», etc.) et la multiplicité/diversité des sujets « producteurs » de données (enseignants, élèves, observateurs, parents, etc.),

- la «triangulation » des données, c'est-à-dire leur interprétation en tenant compte à la fois de la diversité des sujets et de la diversité des points de vue, admettre ainsi qu'on ne peut parler d'objectivité dans les faits éducatifs et qu'on doit reconnaître la valeur de tous les points de vue.

Dans ce contexte, le système d'indicateurs a aidé les enseignants à clarifier leurs « problèmes de recherche » et à les mettre en relation entre eux. Dans les études de cas qui ont été produites à la fin de la recherche (17 études de cas, représentatives de presque toutes les écoles qui, en Italie, avaient participé au projet), les enseignants ont décidé d'indiquer, dans la proposition initiale de chaque étude, les «indicateurs explorés » dans leur recherche-action. Il est intéressant de noter que, bien que le système des indicateurs ait été accepté et utilisé, les indices et les variables que nous avions identifiés dans la recherche précédente ont été modifiés et adaptés par chaque enseignant et pour chaque projet. Le système semble donc avoir une validité générale, mais il a besoin d'être adapté à la réalité et à la spécificité des écoles et des projets.

Les indicateurs ont également constitué des éléments sur lesquels fonder l'évaluation des projets, évaluation participative basée sur la discussion et la comparaison des résultats et études de cas par tous les participants, enseignants et «facilitateurs ». Le processus utilisé pour valider les données a été fondé sur une triangulation de données (recueillies en utilisant des techniques et points de vue différents) et une triangulation d'interprétations (enseignant qui conduisait l'action - observateur externe - groupe).

L'évaluation du processus de la recherche ENSI, tant en Italie que dans d'autres pays, a été pertinente pour les enseignants eux-mêmes. Ils ont pu modifier rapidement leurs techniques, leurs méthodologies, et leurs objectifs, et utiliser les données de la recherche pour une action de plus en plus efficace. L'ENSI a mis en évidence les différences entre ce processus et celui plus répandu de la réflexion individuelle, plus informelle, moins structurée, et sans confrontation avec d'autres points de vue. Le résultat est encore une auto-évaluation, mais elle est rendue acceptable pour le décideur puisqu'elle a fait l'objet de discussion de la part du conseiller pédagogique et du groupe. De plus, cette évaluation était une forme de responsabilisation et de contrôle de qualité acceptés par les écoles.

Des systèmes d'indicateurs ont également été utilisés dans d'autres pays, surtout pour évaluer des projets et des activités d'ErE entreprises par les écoles, mais aussi pour l'évaluation de programmes proposés par des associations ou des centres d'ErE.

Éducation relative à l'environnement, Volume 2 | 2000 

la municipalité de Séville a publié, en collaboration avec le Département d'Éducation et de Science du Conseil d'Andalousie, un "Catalogue de critères pour l'évaluation des programmes d'éducation relative à l'environnement» (Centro Municipal de Investigacion, 1992). La proposition s'adressait surtout à des éducateurs professionnels de l'ErE. Bien que son objectif principal était avant tout de repérer des critères, plutôt que de définir un système où les relations sont mises en évidence, les aspects communs avec des indicateurs de qualité sont nombreux. Les critères sont construits sur base d'une conception de l'ErE définie à partir de documents internationaux, dont les principes sont illustrés au début du document. Ils sont proposés non seulement comme instrument d'évaluation, mais aussi comme instrument de soutien et de planification. Ces critères se réfèrent :

- au projet, critères généraux : buts, attitudes et comportements ;

- au programme de l'activité : objectifs, méthodologie et contenus ;

- aux ressources: élaboration de documents et de matériel de soutien, utilisation des ressources matérielles à disposition ;

- au personnel responsable de la réalisation du programme ;

- aux structures des centres d'ErE ;

- à l'évaluation.

méthodologie proposée pour une auto-évaluation en utilisant ces critères a comme points de référence explicites l'évaluation « démocratique », dans le sens de MacDonald (1974), et l'évaluation formative qui, à travers des méthodologies de type qualitatif et ethnographique, fait également appel à la triangulation et donc a recours à une équipe d'évaluation externe.

41 Un autre exemple vient des Pays-Bas où, ces dernières années, on a tenté de concilier les processus d'évaluation de qualité, avec les exigences de l'ErE ou de l'éducation au développement durable. Un exemple de cette tendance, où la poussée du marché vers une qualité se conjugue avec un approfondissement des valeurs propres à l'ErE, est fourni par le travail du SLO (Institut Néerlandais pour le développement des curricula). Celui-ci a proposé à certains établissements pilotes un projet sur les indicateurs de qualité. Le Groupe de travail, composé de chercheurs du SLO, des proviseurs et coordinateurs d'ErE des établissements impliqués et des chercheurs d'une agence «Impulse» - pour le «management» éducatif, s'est fixé comme objectif de "développer un ensemble d'indicateurs et de stratégies utilisables par des écoles qui veulent améliorer leur qualité par rapport au développement soutenable, dans ses aspects pédagogiques, éducatifs et de gestion ». Ce groupe a essayé d'identifier quelles «caractéristiques les activités dans le domaine de l'ErE doivent avoir pour contribuer aux critères de qualité que l'école se fixe " (Ankoné et coll., 1998, p. 2).

Pour pouvoir atteindre cet objectif le groupe s'est interrogé sur le rapport entre indicateurs de qualité pour l'ErE et ceux qui peuvent être trouvés pour l'école. Lorsque le modèle et la liste des indicateurs ont été structurés, il a fallu vérifier leur utilité dans la pratique quotidienne. Le modèle proposé par le groupe de travail prévoit trois catégories d'indicateurs de qualité (figure 3):

- rapport aux contenus de l'ErE ;

- rapport aux relations avec l'extérieur ;

- rapport au système de « soin » pour l'environnement à l'école (Ankoné et coll., 1998, p. 3). 


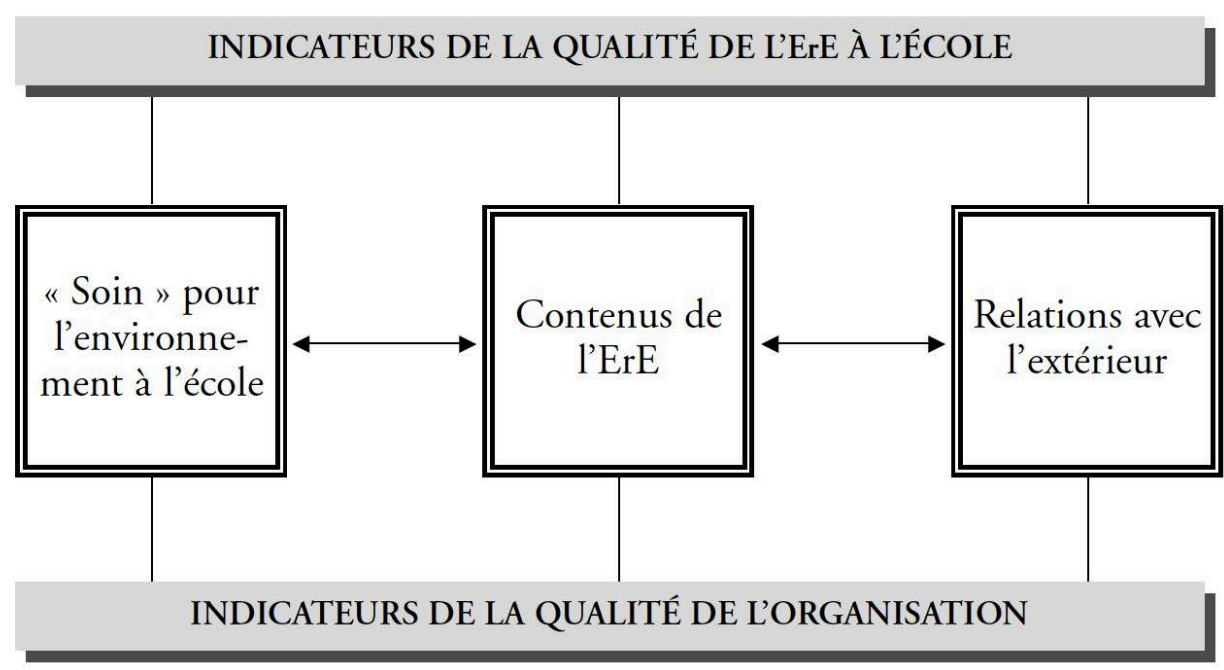

Figure 3 : Le système proposé par le SLO définition d'éducation à la durabilité est reprise dans le rapport Brundtland; dans un autre document de Pieters et coll. (1998) on propose une conceptualisation plus élaborée des caractéristiques envisagées pour l'ErE), mais il n'explicite pas la méthodologie et le paradigme de référence pour l'auto-évaluation que l'école devrait entreprendre. Néanmoins, l'idée que les avis doivent être négociés et concertés entre 
les sujets et qu'il n'existe pas de standards prédéfinis, va dans le sens de l'utilisation des indicateurs dans une perspective critique et formative.

\section{Une clé de lecture des projets} qui a orienté les projets et sur laquelle ont été articulés les Rapports Nationaux des 19 pays participants. Toutefois, les indicateurs définis au début sont-ils, a posteriori, suffisants pour décrire la richesse des processus mis en place? En lisant les Rapports Nationaux, en analysant les études approfondies de cinq des pays participants, et les documents des quatre Séminaires Internationaux organisés, de nouveaux éléments émergent. Ils constituent la «valeur ajoutée » du projet, en termes de connaissances et de conscience (Mayer, 1995):

- À côté des qualités dynamiques des élèves, le projet a fait émerger les qualités dynamiques des enseignants, il a créé des contextes qui les ont amenés à réfléchir à leurs méthodes et à les modifier. Si les enseignants ne font pas preuve d'indépendance à l'égard de la tradition, s'ils manquent de souplesse, s'ils ne se livrent pas à une réflexion critique et ne se mettent pas à l'écoute des autres, ils ne peuvent transmettre ces qualités à leurs élèves.

- Les valeurs, et le rapport entre valeurs éducatives et environnementales, sont apparus comme une question fondamentale pour l'ErE : comment expliciter ses propres valeurs et respecter celles des autres ? La difficulté à laquelle l'enseignant se heurte lorsqu'il travaille en ErE, comme dans toute autre éducation associée aux valeurs, est de " croire à ce que l'on fait tout en donnant l'occasion à d'autres croyances de s'exprimer » (Caiani, enseignante italienne, dans Losito et Mayer, 1995, p. 66).

- La question des valeurs est liée à une nouvelle idée du savoir, où le terme émergent est celui de complexité, où les relations sont aussi importantes, voire plus, que les objets qui font partie du système, où on ne peut plus prétendre à la supériorité du savoir objectif et disciplinaire, où on tient compte des composantes affectives et du système de valeurs dans la construction des connaissances : « Nous ne pouvons jamais identifier la manière dont sont les choses, surtout lorsqu'il s'agit d'êtres humains et de leur milieu, sans déjà interpréter ce

Éducation relative à l'environnement, Volume 2 | 2000 
que nous trouvons, nous préparant implicitement à prendre des décisions ou à porter des jugements de valeur » (Stengers, 1992).

- Les processus qui contribuent à créer une culture de la complexité doivent, de même, prendre en compte le risque et l'imprévisibilité de toute situation, éducative et environnementale : il ne s'agit plus pour l'enseignant d'avoir confiance en un contenu - des règles établies, des donnés, des méthodes -, mais confiance en des processus qui nous permettent, non pas d'éliminer l'erreur et les conflits, mais de les maîtriser.

51 Ces qualités émergentes constituent de nouveaux indicateurs qui s'ajoutent aux premiers pour tracer une carte plus riche et complexe que celle construite au début, une carte qui laisse de la place aux produits et aux processus, dans laquelle nous retrouvons les résultats relatifs aux objectifs que nous poursuivons, mais aussi des idées nouvelles et, en partie, imprévisibles que le projet a contribué à faire naître et à mettre en évidence.

Cette émergence d'éléments inattendus est, d'autre part, une caractéristique du paradigme sociocritique (Flogaitis et Liarakou, 2000). Si on refuse une étroite relation entre cause et effet, et on étend donc l'évaluation à tous les champs, si on refuse, en outre, la position plus exagérément relativiste, une des responsabilités de l'évaluateur est de faire émerger résultats et éléments de réflexion implicites et non prévus. Ceci mettra, dès lors, en évidence la spécificité et l'originalité de chaque projet, au-delà des objectifs qui avaient été définis.

Un exemple d'attention aux « émergences » dans les projets internationaux se retrouve dans le Rapport d'évaluation d'un Projet du WWF, «Forêts Méditerranéennes » (Mayer, 1997). La demande d'évaluation concernait essentiellement les matériaux produits, leur expérimentation dans les classes et, en deuxième plan seulement, l'évaluation de la contribution de la composante internationale du projet. L'évaluation a fait émerger que la dimension internationale constituait l'élément le plus intéressant, mais aussi le plus problématique du projet. Essayer de produire le même matériel pour des cultures différentes - bien que proches - avec différentes représentations de ce que sont l'école, l'éducation et l'ErE, veut dire accepter le conflit et éviter de le résoudre à travers une simple juxtaposition d'idées et de matériaux ou pire, à travers la prédominance d'un point de vue sur les autres. La décision opératoire prise par les différents bureaux nationaux du WWF (Espagne, Italie et Grèce) fut à cette époque celle de produire un matériel souvent identique à la base, mais avec de consistantes options nationales. Toutefois, la contribution principale de l'évaluation externe fut celle d'avoir mis en évidence un indicateur négligé : la valeur des diversités nationales, en particulier des «différentes conceptions de forêt méditerranéenne et d'ErE, des différents systèmes éducatifs, des différents rapports que les WWF nationaux avaient localement établis avec les Autorités éducatives » (Mayer, 1997).

\section{Conclusion}

Les exemples proposés montrent que l'utilisation d'indicateurs de qualité peut constituer une stratégie cohérente avec une vision sociocritique de l'ErE et de l'évaluation. Toutefois, pour arriver à cette cohérence, il faut insister sur les valeurs de départ, spécifier l'utilisation des termes et surtout définir le type de procédure à suivre. 

définition de qualité. Cela ne veut pas dire qu'il n'est pas possible, selon les projets et les contextes, de définir des indicateurs, mais qu'ils ne peuvent être réduits ni à des " produits finaux qui s'adaptent à des standards fonctionnels prédéfinis » (Elliott, 1991, p. 123) ni à des «piquets signalétiques préfixés » (Elliott, 1993, p. 62). Leur utilisation n'est donc pas utile à un «contrôle de qualité » dans la logique du marché, mais à une amélioration de la qualité, à travers la construction de connaissances partagées, utilisables pour le développement et le changement des pratiques éducatives. évaluation qui s'inspire du paradigme sociocritique, et dans ce contexte proposer un système d'indicateurs signifie proposer une conception de l'ErE et de son rôle comme facteur de changement à l'intérieur des processus éducatifs, de l'organisation scolaire, de la société entière. Un système est donc toujours, au moins en partie, construit sur base du système de valeurs et du modèle d'ErE de référence. Celui-ci propose pourtant un ensemble de directions à suivre et auxquelles on sera confronté durant le déroulement du projet. Le système est, néanmoins, une construction a posteriori, pour pouvoir décrire les éléments émergents, les caractéristiques non prévues, que les projets ont contribué à rendre explicites et à construire. Les deux modalités, les deux cartes, se complètent mutuellement, et permettent de reconstruire le parcours complet, en gardant trace soit du processus soit du produit.

Mais l'élément qui valorise l'utilisation des indicateurs dans le paradigme sociocritique par rapport à son utilisation dans le paradigme positiviste est donné par les modalités nécessaires pour sa construction:

- Le système d'indicateurs ne peut naitre de la seule réflexion et de la confrontation entre tous ceux qui prennent part au Projet : il ne s'agit pas de recueillir les opinions de " clients » ou de " consommateurs », mais d'impliquer toutes les parties pour qu'elles se transforment en vrais partenaires du projet (Piette et coll., 2000).

- La définition d'un modèle de référence, la délimitation des aires d'attention, la construction des indicateurs pour chaque aire doivent constituer les éléments d'une réflexion commune et d'une négociation, élargie aux commettants, au public, aux opérateurs, même extérieurs au projet d'ErE spécifique que l'on veut développer. La définition des indices, des éléments observables, doit émaner d'une concertation surtout avec ceux qui prennent une partie active dans le projet. C'est seulement ainsi que, partant d'un système de contrôle imposé, l'évaluation se transforme en un système de réflexion collective, qui tient compte des diversités et des spécificités de chaque contexte.

- En toute cohérence, et compte tenu que « la qualité de l'éducation peut être décrite et jugée, mais non pas standardisée ou mesurée » (Elliott, 1991, p.151), on ne peut assigner des standards à des indicateurs précis, mais seulement définir des seuils, au-delà desquels on ne peut plus parler ni d'ErE ni de qualité.

- Pour éviter l'autoréférence, pour assurer que le processus de réflexion ne se ferme sur soi, pour faire émerger points de force et points de faiblesse implicites, les systèmes d'indicateurs doivent toujours prévoir la présence d'une équipe d'évaluation externe ou, en tout cas, d'un système de triangulation, fondamental pour assurer une confrontation et un débat également utilisables par ceux qui n'ont pas pris part au projet.

Ces combinaisons entre indicateurs de qualité et instruments d'observation, de description qui tiennent compte du caractère qualitatif des processus, entre autodéfinition des indices et définition collective des indicateurs, entre auto-évaluation 
et évaluation participative, entre absence de standards et présence d'une confrontation externe, constituent à mon avis une proposition d'évaluation pour l'ErE. Cette proposition cherche à maintenir un équilibre entre exigence d'évaluation de la qualité et conscience du caractère toujours élusif et en évolution de la qualité elle-même, entre respect de la diversité et exigence d'utiliser cette diversité pour une réflexion collective utile pour le changement de notre système éducatif voire de notre planète.

\section{BIBLIOGRAPHIE}

Ammassari, R. et Palleschi, M.T. (1991). Educazione Ambientale : gli Indicatori di qualità. Isfol SR, Milano : Franco Angeli.

Ankoné, H., Kuypers, B., Pieters, M., Van Rossum, J. (1998). Quality Indicators for Environmental Education. Enschede, Hollande : National Institute for Curriculum Development (SLO).

Bottani, N. (1989). Il progetto CERI/OCDE sugli indicatori internazionali dell'istruzione. Scuola Democratica, 12(1-2), 4-12.

Centro Municipal de Investigacion y Dinamizacion Educativa. (1992). Catalogo de criterios para la evaluaciòn de programas de Educaciòn Ambiental. Ayuntamiento de Sevilla, Aera de Cultura y Educaciòn.

CERI/INES. (1989). Indicateurs internationaux de l'enseignement : éclairages conceptuels. Rapport 89.07. Paris : CERIS/INES.

Eide, K. (1989). The Need for Statistical Indicators in Education. OECD Washington Conference on Educational Indicators. Scuola Democratica, 12(1-2), 87-93.

Elliott, J. (1991). Action Research for Educational Change. Milton Keynes, Philadelphia : Open University Press.

Elliott, J. (1993). Reconstructing Teacher Education : Teacher Development. London. Washington DC. : The Falmer Press.

Elliott, J. (1995). ENSI, recherche action et perfectionnement des enseignants. Environmental Learning for the XXI ${ }^{\circ}$ Century. Paris : OECD Documents.

Gardner, (1983). A Nation at Risk : the Imperative for Educational Reform. Washington, D.C. : Gouvernemental Printing Office.

Ginzburg, C. (1986). Miti, emblemi, spie. Torino : Einaudi.

Guba, E. et Lincoln, Y. (1989). Fourth Generation Evaluation. Newbury Park : Sage Publications.

Kelley Lainé, K. (1990). The Environment at School. The OECD Observer, 13-16. Liarakou, G. et Flogaitis, E. (2000). Quelle évaluation pour quelle éducation relative à l'environnement ? Éducation relative à l'environnement : Regards, Recherches, Réflexions, 2.

Losito, B. et Mayer, M. (1995). Educazione Ambientale : un banco di prova per l'innovazione. (version en anglais Environmental education : a challenge for educational innovation). Rapporto Nazionale Italiano per la ricerca ENSI. Frascati : CEDE. 
MacDonald, B. (1974). Evaluation and the Control of Education. Dans B. MacDonald et R. Walker (dir.), Innovation, Evaluation, Research and the Problem of Control (SAFARI). Norwich : Centre for Applied Research in Education.

Mayer, M. et Cogliati Dezza, V. (1991). Évaluer la qualité des projets d'éducation à l'environnement : un système d'indicateurs. Dans A. Giordan, J.L. Martinand et C. Souchon (dir.), École et média face aux défis de l'environnement. XIIIes Journées Internationales de Chamonix.

Mayer, M. (1991). Environmental Education in Italy : Proposals for an Evaluation Strategy. European Journal of Education, 26(4).

Mayer, M. (1994). L'action de l'école en faveur de l'environnement : évaluation des résultats. Evaluating Innovation in Environmental Education. Paris : OECD Documents.

Mayer, M. (1995). Quality Indicators and Innovation in Environmental Education. L'éducation à l'environnement pour le XXIe siècle. Paris : OECD Documents.

Mayer, M. (1997). The WWF Project Mediterranean Forests. Evaluation Report. Frascati : CEDE.

Mayer, M. (1999). Éducation relative à l'environnement et recherche-action. Éducation relative à l'environnement : Regards, Recherches, Réflexions, 1, 195-202.

Norris, N. (1993). Evaluation, Economics and Performance Indicators. Dans J. Elliott (dir.), Reconstructing Teacher Education : Teacher Development. London \& Washington DC. : The Falmer Press. p. 31-38

Nuttal, D. (1992). Les indicateurs internationaux de l'enseignement : leurs fonctions et leurs limites. In OCDE-CERI, L'OCDE et les indicateurs internationaux de l'enseignement. Un cadre d'analyse. Paris : OCDE. p. 15-25.

Oakes, J. (1989). Educational Indicators. A guide for Policymakers. OECD Washington Conference on Educational Indicators. Scuola Democratica, 12(1-2), 56-86.

OCDE-CERI. (1991). Environment, school and active learning. Paris : OCDE. OCDE-CERI. (1992). L'OCDE et les indicateurs internationaux de l'enseignement. Un cadre d'analyse. Paris : OCDE.

OCDE. (1994). Evaluating Innovation in Environmental Education. Paris : OCDE-CERI.

OCDE. (1995). L'éducation à l'environnement pour le XXIe siècle. Paris : OCDE-CERI.

Pieters, M., Broné, M. et Kolker, G. (1998). Conceptualisation of Environmental Education. Enschede, Hollande : National Institute for Curriculum Development (SLO).

Sauvé, L. (1997). Pour une recherche de type critique en éducation relative à l'environnement. Dans C. Baudoux et M. Anadón (dir.), La recherche en éducation, la personne et le changement. Les cahiers du LABRAPS, 23. p. 105-117.

Stake, R. (1980). The Case Study Method in Social Inquiry. Dans H. Simons (dir.), Toward a Science of the Singular. Norwich : University of East Anglia.

Stake, R. (1988). La valutazione « responsive ». In M.L. Giovannini (dir.), La valutazione delle innovazioni nella scuola. Bologna : Cappelli.

Stenhouse, L. (1975). An Introduction to Curriculum Research and Development. London : Heinemann. Stengers, I. (1992). Progrès et complexité : tension entre deux images. Conférence internationale : Immagini della Società, della natura e della scienza attraverso l'educazione ambientale. Perugia (Italia). 


\section{RÉSUMÉS}

Les indicateurs de qualité ont surtout été utilisés dans la recherche éducative internationale dans un paradigme positiviste. Si une telle approche évaluative peut être utile pour l'évaluation des programmes et des projets d'éducation relative à l'environnement (ErE), elle demeure toutefois limitée. Cet article explore les possibilités que les indicateurs de qualité offrent à l'évaluation en ErE, non seulement pour l'évaluation des résultats, mais aussi pour l'orientation et la conduite des projets. Entre autres, les indicateurs peuvent mettre en évidence les caractéristiques «émergentes » et non prévues d'un projet d'ErE. L'auteure insiste sur la prise en compte du caractère qualitatif des processus éducatifs ainsi que des stratégies d'auto-évaluation et d'évaluation participative.

Quality indicators have been adopted in international educational research mainly as tools within a positivist research paradigm. Their approach to evaluation can be seen as consistent with some evaluation needs of Environmental Education (EE) programs and projects. This paper explores the possibilities offered to EE by the use of quality indicators, not only to evaluate results but also to orient and steer the projects. The indicators can also highlight the "emerging" and unexpected characteristics of EE projects. The author insists on taking into consideration the qualitative nature of educational processes together with the strategies of self-evaluation and participative evaluation.

\section{AUTEUR}

\section{MICHELA MAYER}

Physicienne et titulaire d'un doctorat en didactique des sciences, Michela Mayer est chercheure au Centre Européen de l'Éducation, où elle s'occupe de recherche en ErE et d'évaluation. Chargée de la participation italienne au projet ENSI de l'OCDE, membre du comité d'édition de la revue Educational Action Research, elle coordonne actuellement en Italie une recherche évaluative sur les compétences des élèves en matière d'environnement. 